\title{
Bioclimatologie
}

\section{Masse en eau des feuilles: détermination non destructive en milieu naturel}

\author{
S de Parcevaux, JM Bertolini *, N Katerji \\ INRA, Bioclimatologie, F-78850 Thiverval-Grignon, France
}

(Reçu le 10 décembre 1994 ; accepté le 6 novembre 1995)

\begin{abstract}
Résumé - La masse en eau des feuilles peut être déterminée à partir de la cinétique de la température après une modification brutale du bilan d'énergie par un éclairement additionnel. Une étude critique de certains aspects techniques d'une méthode employée précédemment a conduit à des améliorations instrumentales (utilisation des fibres optiques et de capteurs thermiques différentiels sans contact). II en résulte un appareillage portable permettant de travailler tant en conditions contrôlées que naturelles. Les vérifications expérimentales effectuées sur plusieurs espèces à comportement hydrique contrasté ont permis de constater que les mesures de teneur en eau obtenues avec l'appareillage portable conduisent à des résultats proches de ceux déterminés directement par la méthode gravimétrique.
\end{abstract}

cinétique thermique / radiométrie différentielle / tournesol / maïs / tomate

Summary - Mass leaf water content: non-destructive determination in natural conditions. Leaf water mass can be determined from thermal response to abrupt changes in the energy balance. A previous method was improved by using optical fibers and non-intrusive thermal sensors. The new apparatus is portable and well adapted for measurements under both controlled and field conditions. An experimental test with several plant species characterized by contrasting hydric properties showed a good relationship between measurements with the improved instrument and the direct weighing of fresh and dry leaves.

thermal kinetics / differential radiometry / sunflower / maize / tomato

\section{INTRODUCTION}

La teneur en eau des feuilles conditionne leur bon fonctionnement physiologique. II existe une valeur optimale de ce facteur et des valeurs extrêmes au-delà desquelles le fonctionnement est perturbé ou complètement inhibé. ( $C f$ la synthèse bibliographique de Boyer complétée par Roy, 1980.)
Le contrôle de l'état hydrique des plantes est donc un facteur essentiel à la bonne réussite des cultures. L'irrigation permet de maîtriser leur alimentation hydrique. Mais encore faut-il disposer de critères adéquats basés sur des grandeurs caractérisant l'état hydrique des végétaux (Katerji et al, 1988). Les plus pertinentes a priori sont la teneur en eau des feuilles et le potentiel hydrique foliaire. Mais ces grandeurs doivent 
varier avec l'alimentation hydrique de la feuille, ce qui signifie que les réservoirs temporaires d'eau de la plante et les phénomènes de régulation associés à la circulation de l'eau se trouvent dans la feuille ou à sa proximité immédiate. C'est le cas des plantes de type "anisohydrique» ou «labile» (de Parcevaux, 1963). À titre d'exemple, on peut citer la betterave, le tournesol (Buriol et al, 1984b), le soja (Mastrorilli et al, 1993), l'aubergine (Katerji et al, 1990), le sorgho (Baldy et al, 1993), le maïs au stade jeune (Katerji et al, 1994) et adulte (Tardieu et al, 1990). Chez les plantes isohydriques, beaucoup de céréales, la tomate (Katerji et al, 1988)..., l'essentiel du stockage a probablement lieu hors de la feuille. Dans ce cas, la teneur en eau de la feuille est un moins bon ou même un mauvais critère indicateur de l'état hydrique de la plante. En revanche, chez les plantes de type anisohydrique, il est intéressant de déterminer de façon précise la teneur en eau des feuilles dans les conditions naturelles.

Les méthodes classiques de mesure de l'état hydrique de la feuille conduisent généralement à la destruction de l'organe. Des efforts ont été faits pour la mise au point de méthodes «non destructives». Elles utilisent l'absorption de divers rayonnements, la réflexion du rayonnement incident par la surface foliaire, la variation de la conductivité électrique des tissus ou la mesure de la constante diélectrique. Une revue bibliographique a été rapportée par Buriol et al (1984a). Dans ces méthodes, l'étalonnage est souvent délicat, soit parce qu'elles ne sont pas sélectives pour l'eau (rayons bêta et gamma), soit en raison de l'hétérogénéité de la structure du couvert végétal ou de la feuille (ondes électromagnétiques et conductivité électrique), ou d'une mise au point technique particulièrement difficile (constante diélectrique).

C'est pour éviter ces inconvénients que nous avons mis au point (Buriol et al, 1984a, b) une méthode de détermination de la masse en eau d'une feuille, fondée sur la mesure de l'inertie thermique. En effet, selon la théorie (de Parcevaux, 1972a, b ; de Parcevaux et Perrier, 1973), la pente à l'origine d'une cinétique de réchauffement induite par un flux de rayonnement additionnel conduit à la détermination directe de la capacité calorifique surfacique MC de la feuille et donc à sa masse en eau surfacique (fig 1). Cette méthode, non destructive, ne perturbe pratiquement pas la physiologie de la feuille, ni son environnement : le temps de la mesure, une dizaine de secondes, est très inférieur au temps de réaction des stomates, quelques minutes, et la perturbation radiative est analogue à celle du passage d'un nuage devant le soleil. L'élévation locale de température est inférieure à $4^{\circ} \mathrm{C}$.

Jusqu'à présent l'utilisation de capteurs de contact (thermocouples fins dans une pince adaptée) et d'un dispositif d'éclairage un peu

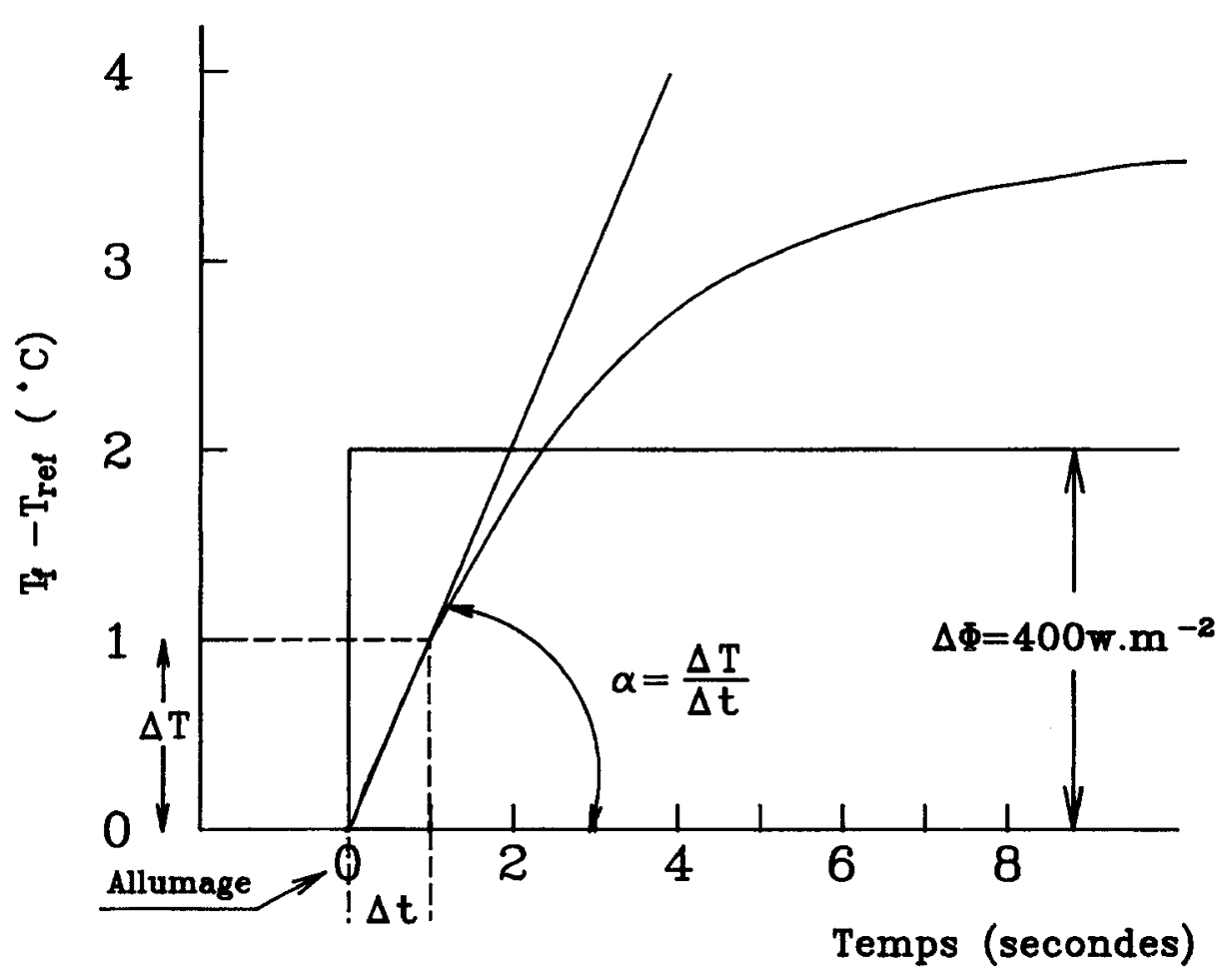

Fig 1. Évolution de la température foliaire en réponse à une variation du rayonnement absorbé (d'après Buriol et al, 1984). 
sommaire alimenté par le secteur introduisait des causes d'imprécision et d'inconfort d'emploi en conditions naturelles, limitant ainsi l'utilisation de ce dispositif aux conditions expérimentales contrôlées. C'est pourquoi nous avons entrepris d'effectuer les améliorations techniques envisagées en 1984 (Buriol et al, 1984a) au niveau des capteurs, de l'automatisation de la mesure, et de l'alimentation électrique.

De façon plus précise, cet article a pour but :

- de présenter un appareillage conçu pour être utilisé dans les conditions naturelles, en changeant les capteurs thermiques, en modifiant le dispositif d'éclairage et en automatisant la mesure ;

- de vérifier l'aptitude de ce dispositif à déterminer la masse en eau surfacique chez les espèces à comportement anisohydrique et même isohydrique.

\section{MATÉRIEL ET MÉTHODES}

Le dispositif (fig 2) est constitué d'un générateur de lumière froide, d'une pince, d'une acquisition de données et d'une alimentation électrique par batterie. La pince (fig 3) supporte la fibre optique et les 2 capteurs infrarouge thermique. Pour réaliser les mesures in situ, l'ensemble du dispositif a été monté sur un chariot présenté figure 4.

La précision de la mesure est limitée par l'hétérogénéité et l'anisotropie éventuelle du matériau, donc par les variations d'épaisseur du limbe dues principalement aux nervures.

\section{Améliorations techniques}

Les principales limitations de la méthode étaient liées aux caractéristiques de l'instrumentation et des capteurs. Les thermocouples utilisés jusqu'à présent étaient des capteurs de contact. Or il n'est pas toujours aisé de réaliser un bon contact thermique entre un thermocouple fin, ayant une inertie minimale, et une feuille dont la surface n'est pas toujours plane, ni même régulière.

\section{Utilisation de capteurs thermiques sans contact}

Les capteurs thermiques sans contact sont basés sur la mesure du rayonnement infrarouge émis par la surface dont on cherche à connaître la température. Ces capteurs sont aujourd'hui disponible de manière courante et relativement bon marché. Nous avons chois des thermophiles miniatures type S25 (Dexter Research : 7300 Huron River Drive, Dexter, MF 48130, États-Unis), chargées au xénon et munies d'une fenêtre transmettant la bande de 8-14 $\mu \mathrm{m}$, dont la constante de temps est de $12 \mathrm{~ms}$ (Amiro et al, 1983 ; Kalma et al, 1988 ; Graham et al, 1989).

Le signal délivré est une tension continue d'une dizaines de millivolts, avec un rapport signal sur bruit du même ordre que celui d'un thermocouple. Le capteur a une impédance de $25 \mathrm{k} \Omega$ avec un NEP de 0,08 $\mathrm{nW} / \mathrm{V} \mathrm{Hz}$; il est compatible avec les entrées bas niveau d'une centrale d'acquisition de données standard. Nous avons utilisé une centrale de type CR10 pilotée par un microprocesseur (Campbell Scientifique Ltd, 14-20 Field Street, Shepshed Leicestershire, LE12 $9 \mathrm{AL}$, Royaume-Uni) qui permet l'acquisition des données, le calcul en temps réel des paramètres et la gestion des automatismes.
Fig 2. Schéma général du dispositif.

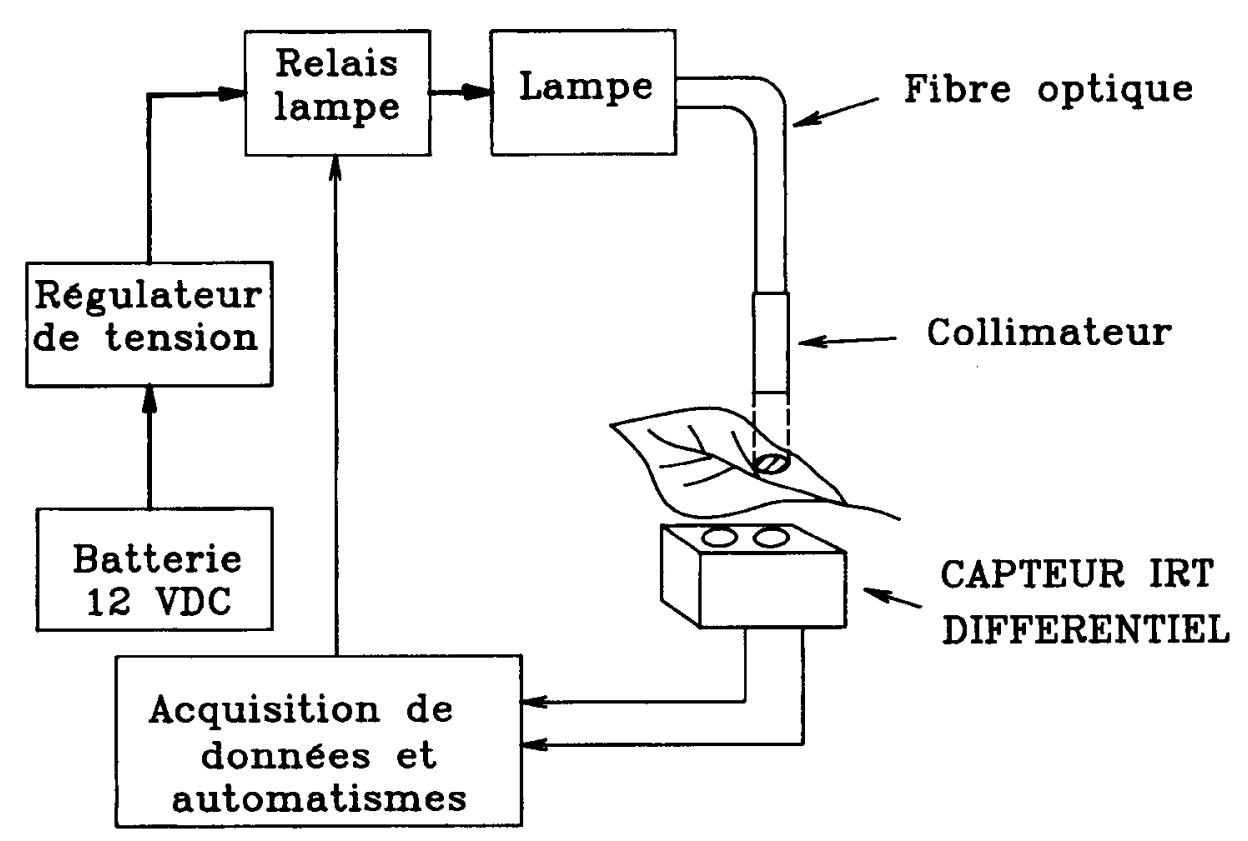




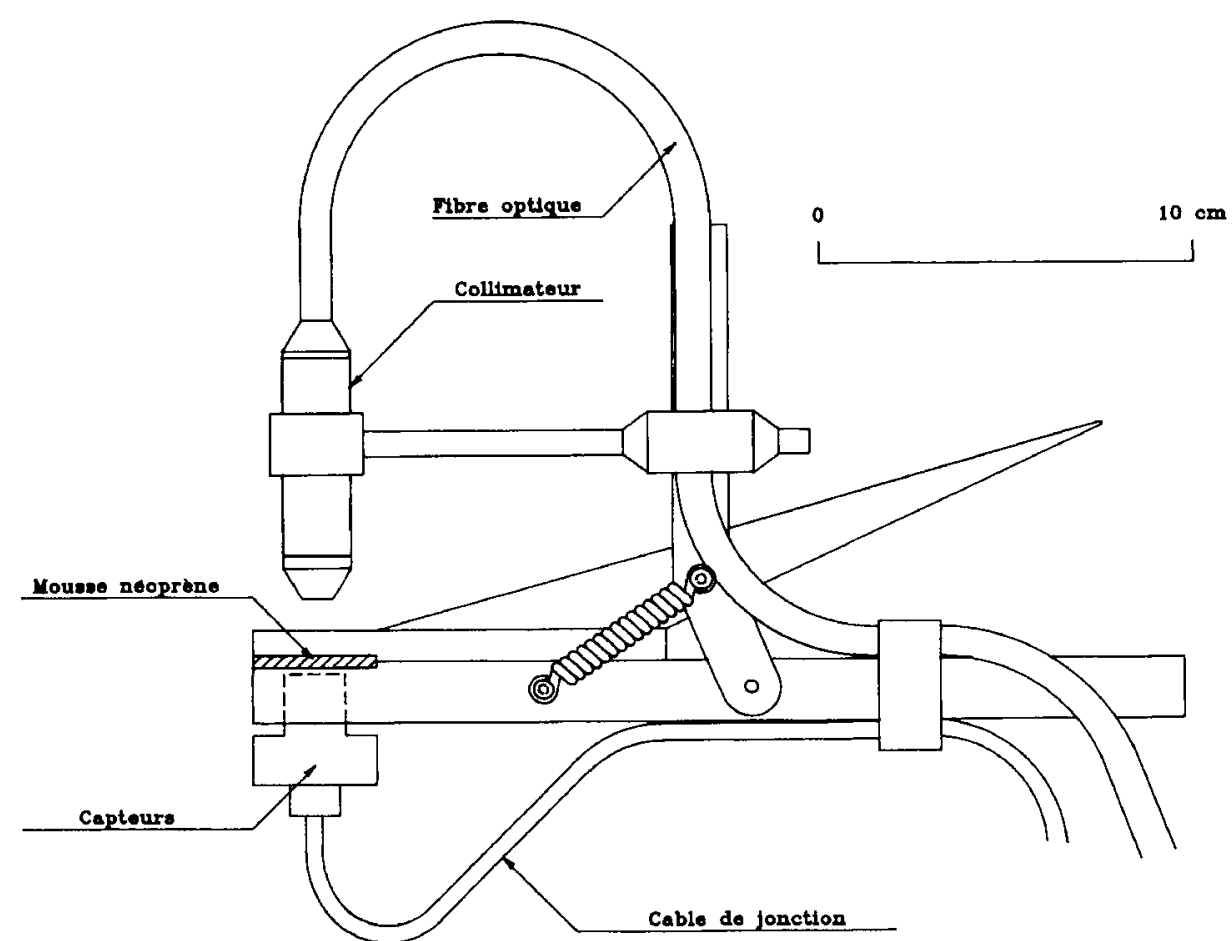

Fig 3. Représentation de la pince de mesure.
Une bonne précision exige une mesure différentielle. Ce qui était facile avec un thermocouple, capteur différentiel par construction, l'était moins avec 2 thermopiles. Un soin particulier a donc été apporté à l'environnement thermique des capteurs. Ils ont été collés avec un adhésif conducteur de la chaleur dans un support en aluminium massif. Deux thermocouples de contrôle ont été préalablement installés, l'un sur le boîtier du capteur, l'autre sur le support en aluminium. Ils permettent de vérifier l'absence de gradient latéral et vertical (fig 5 ).

\section{Utilisation de fibres optiques}

La source de rayonnement additionnel utilisée pour mettre en œuvre la méthode était assez proche de la surface de mesure. La présence de cette source chaude (lampe halogène) était susceptible de perturber les paramètres microclimatiques au voisinage de la feuille (émission de chaleur, rayonnement infrarouge...). Pour éliminer au maximum ces effets indésirables, l'utilisation d'un conducteur de lumière constitué par un faisceau de fibres optiques assurant la transmission du rayonnement additionnel jusqu'à la feuille a permis d'éloigner la source. Le faisceau de sortie de fibres présente un angle d'ouverture de $120^{\circ}$. Un collimateur de $10 \mathrm{~mm}$ de diamètre permet de réduire cet angle à $6^{\circ}$. Les études préalables de Santibanez (1974) ont mis en évidence la nécessité de l'utilisation de rayonnement visible à l'exclusion du rayonnement infrarouge (1 200-2 $500 \mathrm{~nm}$ ). En effet l'étude des propriétés optiques des feuilles montre que d'une part l'absorption du rayonnement est maximale dans le visible, particulièrement dans le bleu et le rouge, et que d'autre part elle est beaucoup plus faible dans le proche infrarouge et dépendante de la teneur en eau. Le filtre anti- calorique utilisé permet de limiter au domaine visible la répartition spectrale de l'énergie additionnelle reçue par la feuille. La tache de lumière obtenue à une distance de $5 \mathrm{~cm}$ de l'extrémité du collimateur a un diamètre de $16 \mathrm{~mm}$ avec une densité de flux de 400 watts par mètre carré environ.

\section{Automatisation de la mise en œuvre}

Une lampe halogène est alimentée sous une tension stabilisée de $8,0 \pm 0,05 \mathrm{~V}$, grâce à une batterie de 12 volts (type automobile) associée à un régulateur de tension. Les mesures, le calcul en temps réel et l'automatisation sont assurés par la centrale CR10 de Campbell. L'ensemble est conçu de façon à être facilement transportable au champ. Le dispositif est cadencé à une fréquence de 8 mesures par seconde.

Selon la théorie (de Parcevaux et Perrier, 1973), la pente à l'origine $\alpha$ est égale au rapport entre l'intensité du flux lumineux additionnel et la masse thermique MC du limbe:

$$
\alpha=\Delta \Phi / M C
$$

Selon la courbe théorique, la pente est maximale à l'origine $(t=0)$. En fait, pendant la première demiseconde, elle reste voisine de 0 . Ce temps correspond à la transmission à travers la feuille de l'onde thermique provoquée par l'impact du rayonnement additionnel. Ce temps de latence est fonction de l'épaisseur et des caractéristiques thermiques de la feuille. C'est ensuite que la cinétique démarre. La figure 6 présente 3 courbes expérimentales obtenues sur des feuilles de tomate plus ou moins riches en eau.

En pratique on estime la pente $\alpha_{0}$ en calculant la droite de régression sur 6 points consécutifs en partant 
Fig 4. Représentation du dispositif mobile pour la réalisation des mesures au champ.

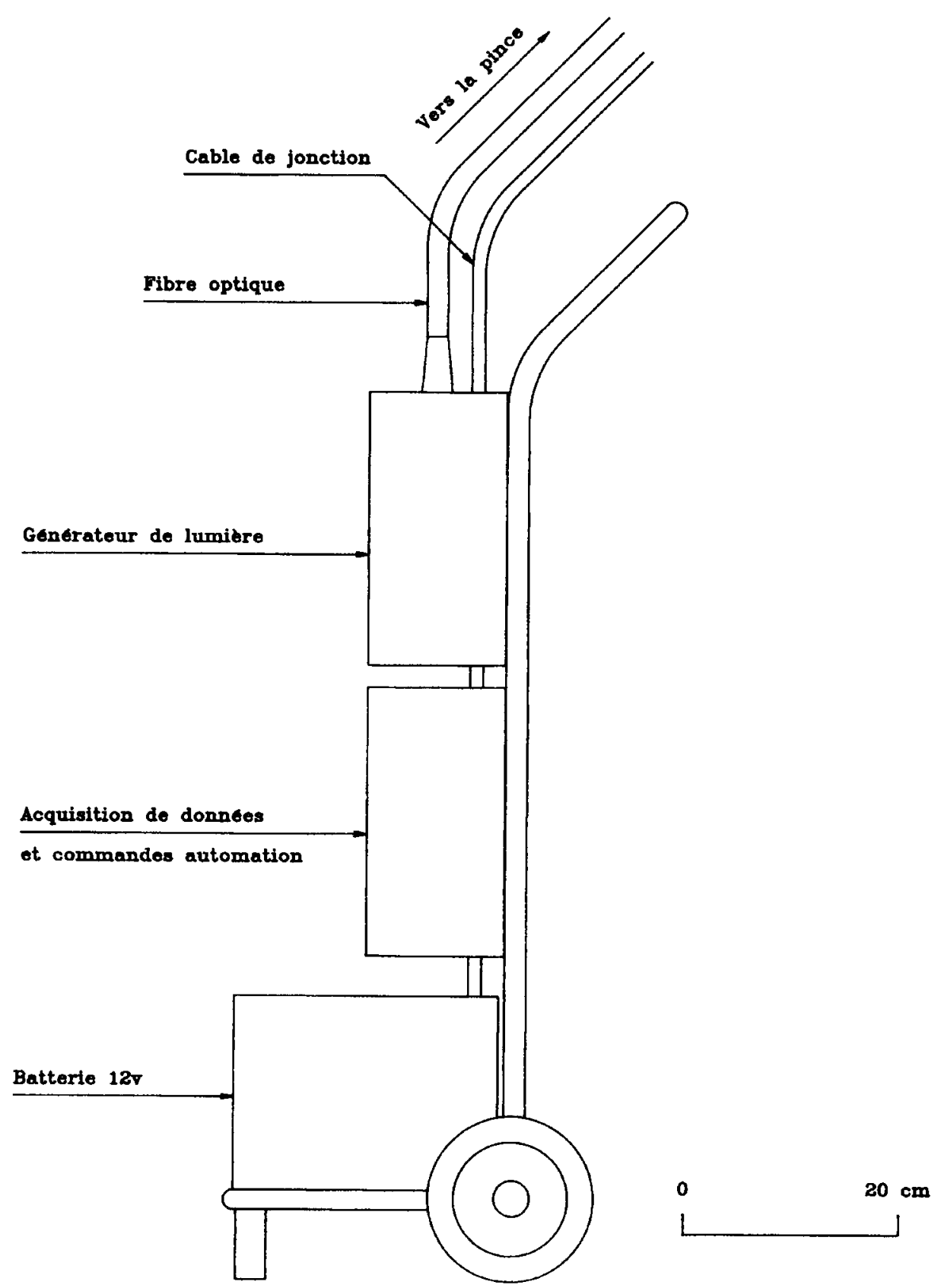

de l'origine des temps ; on réitère le calcul en décalant d'un point jusqu'à ce que la valeur $\alpha_{i}$ de la pente commence à décroître. La valeur maximale recherchée est alors $\alpha_{i-1}$. Ce calcul est réalisé a posteriori et sera intégré au dispositif dans l'avenir. La figure 7 montre l'évolution de la pente $\alpha$ pour une feuille en cours de déshydratation $\left(10 \mathrm{mg} \mathrm{cm}^{-2}\right)$. La valeur maximale de la pente est obtenue pour les mesures comprises approximativement entre 0,5 et $1,5 \mathrm{~s}$ après le début de l'éclairement du rayonnement additionnel).

\section{Étalonnage des capteurs thermiques}

Pour l'étalonnage, le bloc contenant les 2 capteurs a été placé dans une enceinte dont la température peut être régulée entre $5^{\circ} \mathrm{C}$ et $25^{\circ} \mathrm{C}$. La feuille est remplacée par un corps noir dont la température peut être régulée entre $5^{\circ} \mathrm{C}$ et $39^{\circ} \mathrm{C}$ (fig 5). Les conditions de distance et de champ de vision sont identiques à celles utilisées dans les conditions réelles de mesure.

Le signal du capteur peut être assimilé à une fonction linéaire de la différence de température entre la cible et celle du capteur lui-même. Les coefficients d'étalonnage de chaque capteur sont introduits ensuite dans l'acquisition de données.

Les capteurs ont aussi été étalonnés en conditions de variation thermique rapide, l'ensemble variant de 5 à $25^{\circ} \mathrm{C}$ pour une température de corps noir stable de $29^{\circ} \mathrm{C}$. Nous avons vérifié qu'une variation rapide de température $\left(3^{\circ} \mathrm{C} / \mathrm{mn}\right)$ affecte peu le comportement différentiel de l'ensemble des 2 capteurs. 


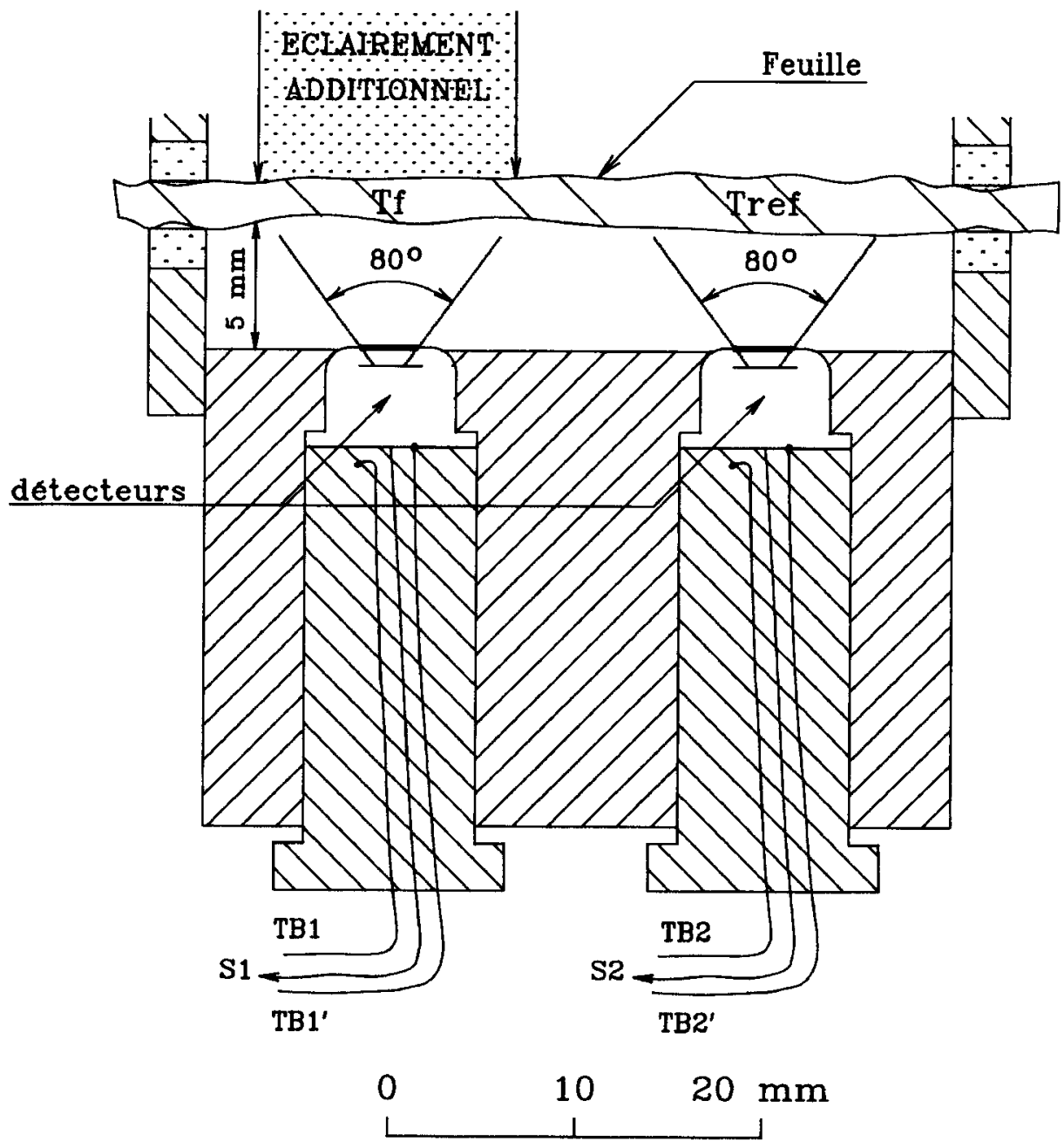

Fig 5. Capteur IRT différentiel composé de 2 capteurs S25 de Dexter Research. TB 1 et 2 , $1^{\prime}$ et $2^{\prime}$ sont des thermocouples de contrôle. $S$ et $S^{\prime \prime}$ correspondent aux sorties des signaux des capteurs.

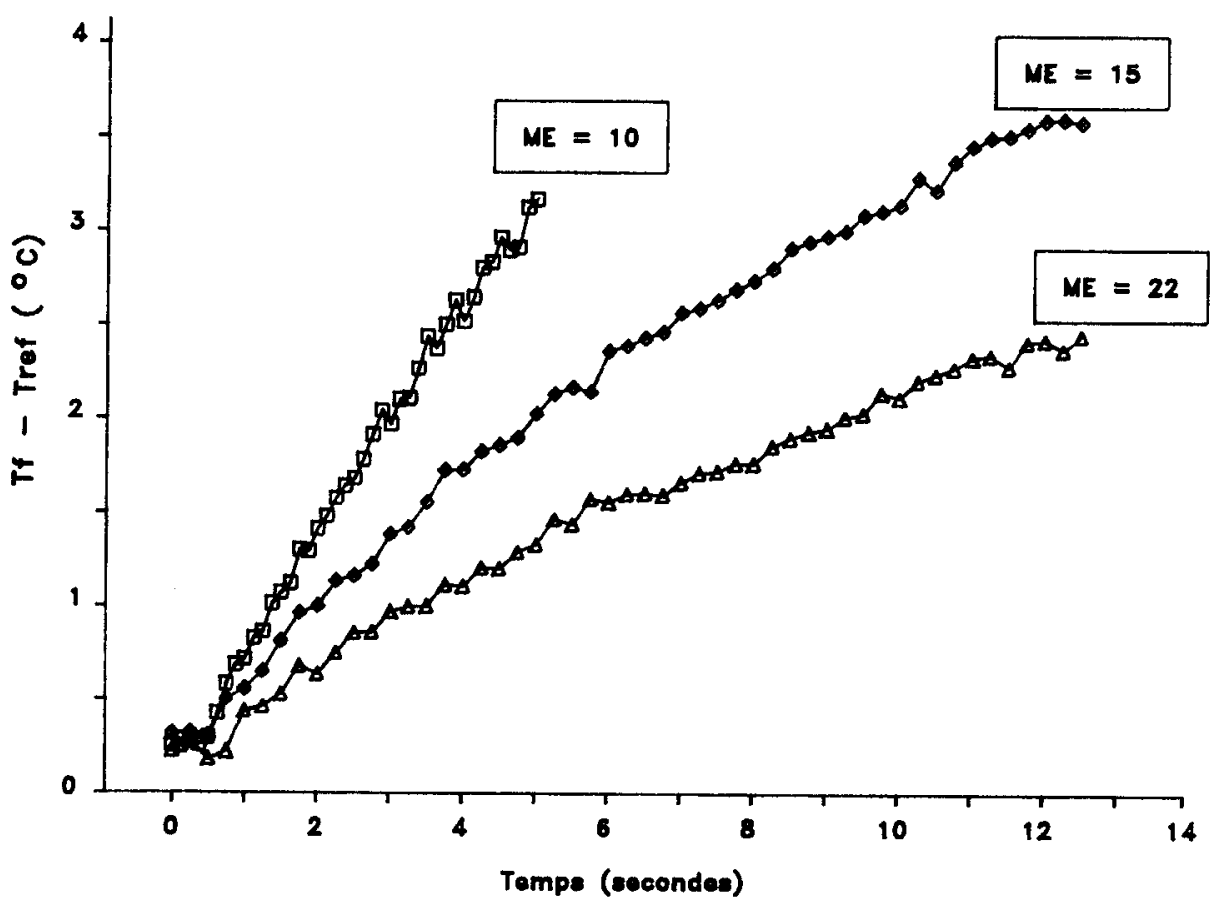

Fig 6. Pentes à l'origine pour 3 feuilles de tomate plus ou moins hydratées. 


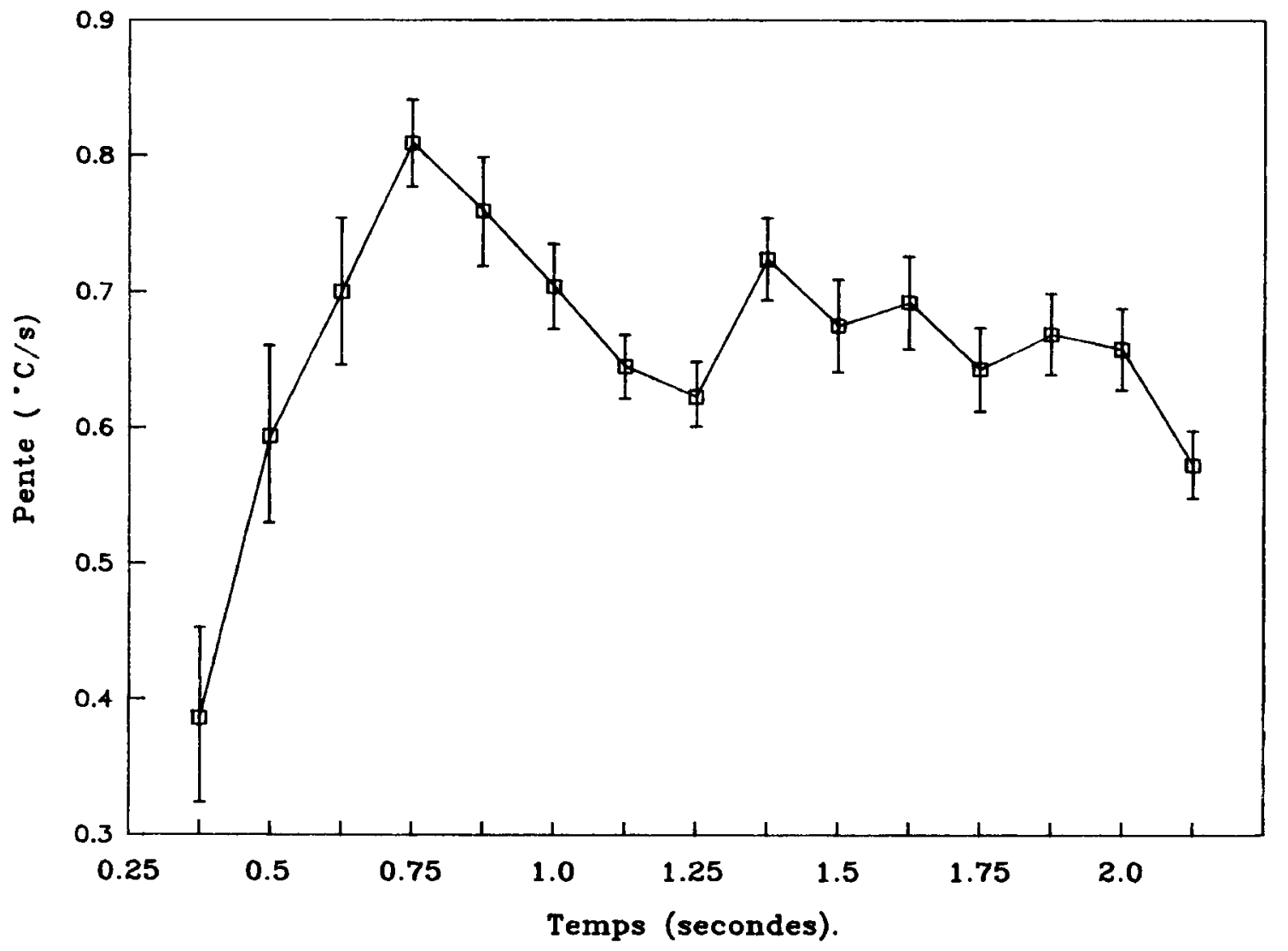

Fig 7. Évolution de la pente glissante mesurée sur 6 points consécutifs en fonction du temps pour une feuille de tomate légèrement déshydratée $\left(10 \mathrm{mg} / \mathrm{cm}^{2}\right)$. La barre verticale représente l'intervalle de confiance à $5 \%$.

\section{Influence de la longueur d'onde du rayonnement utilisé}

Il est important que le rayonnement additionnel absorbé par la feuille reste aussi constant que possible. II faut rechercher un domaine spectral dans lequel l'absorptance est aussi indépendante que possible de l'état physiologique de la feuille, de son épaisseur, de sa couleur et de son état hydrique. Ce domaine est tout d'abord le bleu, mais il est encore difficile d'avoir une source suffisamment puissante dans cette gamme de longueur d'onde. C'est ensuite le rouge. Les variations de couleur verte ne sont généralement pas très rapide ; il est possible d'utiliser l'ensemble du spectre visible, mais il est essentiel d'éliminer le proche infrarouge par un filtre approprié.

\section{Protocole expérimental pour l'étalonnage}

Au laboratoire, le poids d'une feuille entière ou d'un morceau de feuille est suivi au cours du temps. La méthode du choc radiatif est appliquée sur le même échantillon avec 6 à 8 répétitions. À la fin de l'expérience le poids sec de l'échantillon est déterminé par passage à l'étuve, $48 \mathrm{~h}$ à $80^{\circ} \mathrm{C}$.
Au champ, après chaque mesure par choc radiatif, on prélève un disque de feuille correspondant à la zone mesurée que l'on introduit aussitôt dans un flacon hermétique. Chaque échantillon est ensuite pesé à l'état frais et après séchage à l'étuve au laboratoire pour contrôle.

Les mesures ont porté sur des végétaux, soit isohydriques, comme la tomate et le maïs, soit anisohydriques comme le tournesol.

\section{RÉSULTATS}

Sur les figures 8, 9, 10, ME mesurée désigne la masse en eau mesurée selon la méthode pondérale classique qui sert de référence et $\mathrm{ME}$ calculée, la masse en eau déterminée par la méthode du choc radiatif expérimentée ; les valeurs sont exprimées en $\mathrm{mg} / \mathrm{cm}^{2}$.

\section{Plantes isohydriques : maïs et tomate}

La figure 8 présente les résultats obtenus sur du maïs au champ et en serre, en 1992 et en 1993. 


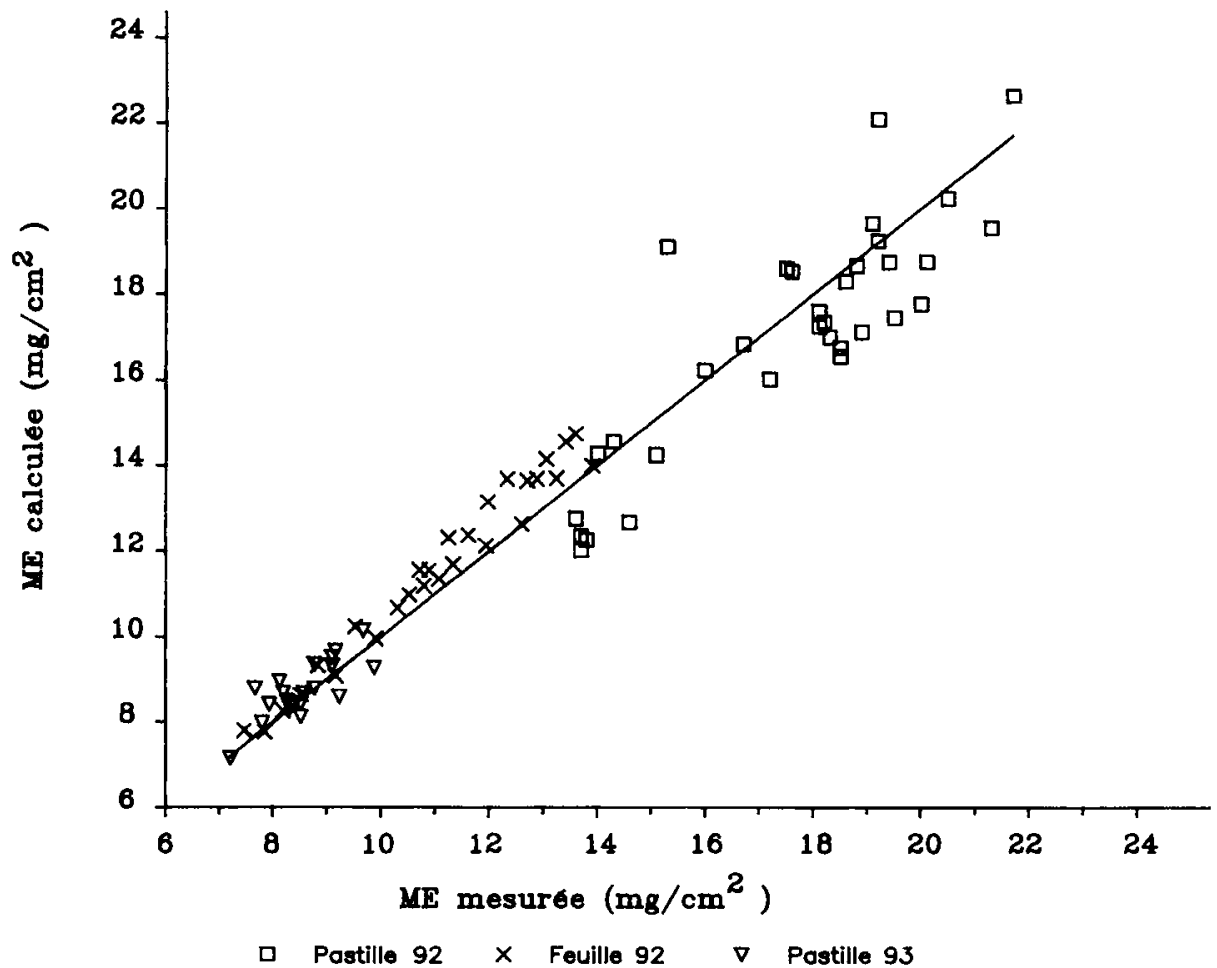

Fig 8. Comparaison des masses en eau foliaire mesurées et calculées pour le maïs au champ et au laboratoire. Les carrés correspondent à des mesures réalisées au champ en 1992 sur des pastilles prélevées sur des feuilles de l'épi ou du sommet de la plante : les échantillons étaient transportés au laboratoire pour pesée dans des conteneurs hermétiques. Les croix correspondent à des mesures réalisées sur des feuilles jeunes entières, mais d'au moins $8 \mathrm{~cm}$ de large, ayant poussé en serre aux mois d'août et septembre 1992. Les triangles renversés correspondent à des mesures sur des pastilles prélevées sur des feuilles de plantes jeunes en serre en juin 1993
L'accord entre les 2 méthodes est satisfaisant. L'erreur reste inférieure à $2 \mathrm{mg} / \mathrm{cm}^{2}$.

La figure 9 présente les résultats obtenus sur tomate en 1992 en serre. L'accord est particulièrement bon dans le cas de la tomate. L'erreur moyenne est de l'ordre de $1 \mathrm{mg} / \mathrm{cm}^{2}$. Les nervures de la feuille de tomate sont régulières et relativement peu développées.

\section{Plante anisohydrique : tournesol}

La figure 10 présente les résultats obtenus sur le tournesol en 1992 au champ. La variabilité dans ce cas est plus grande. Ce résultat, déjà observé sur la même espèce dans une précédente étude (Santibanez, 1974), a été interprété comme une conséquence de l'hétérogénéité de l'épaisseur

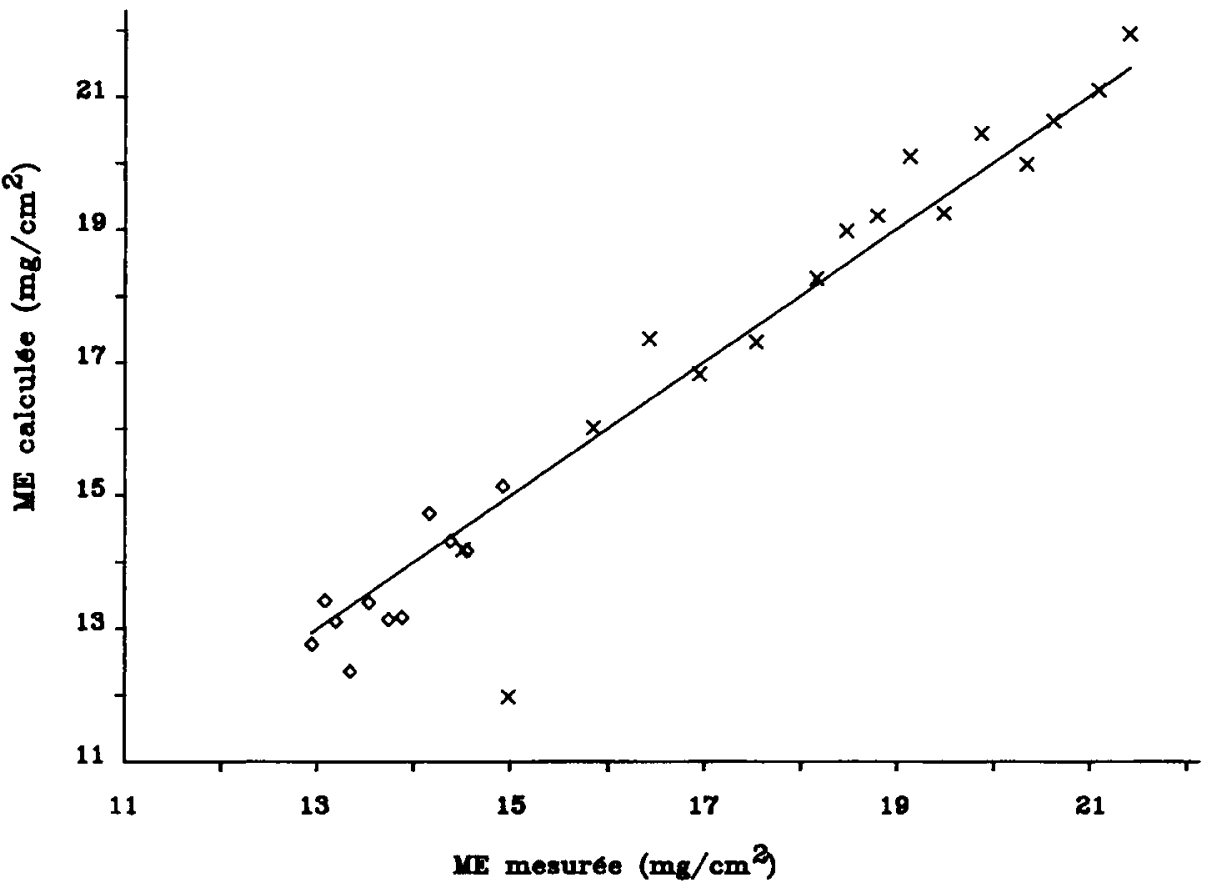

Fig 9. Comparaison des masses en eau foliaire mesurées et calculées pour la tomate au laboratoire. Les symboles $\vartheta$ correspondent à des mesures effectuées par temps ensoleillé en serre. Les symboles $x$ correspondent à des mesures sur des feuilles après une nuit de saturation en sac étanche de plastique. 
Fig 10. Comparaison des masses en eau foliaire mesurées et calculées pour le tournesol au champ avec classement des points de mesure en fonction du rayonnement absorbé calculé (Rg en $W / m 2)$.

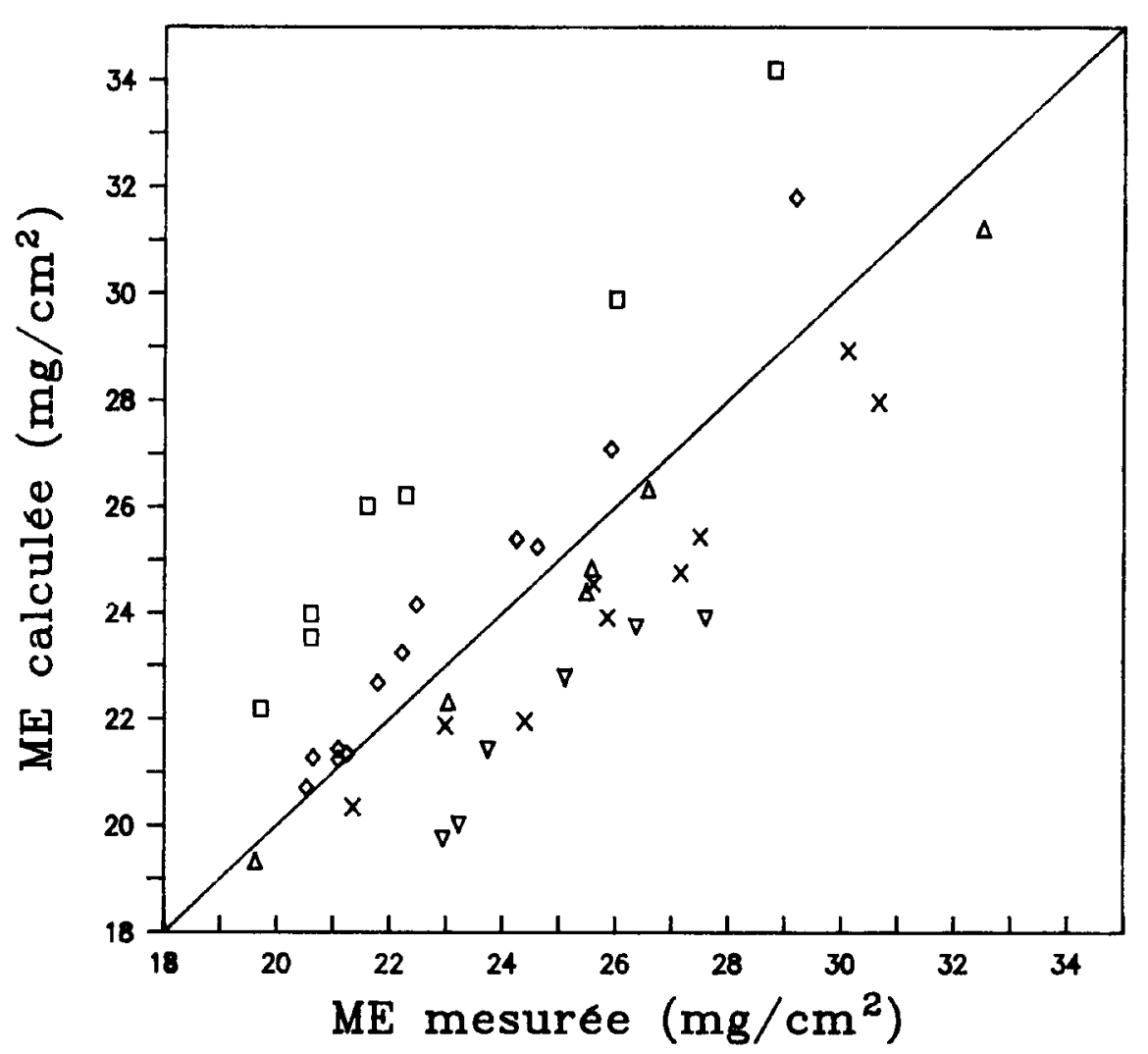

$\begin{array}{lllllllll}R g<400 & \diamond & 400<R g<450 \quad \Delta \quad 450<R g<470 \quad \times \quad 470<R g<500 \quad \nabla & R g>500\end{array}$ liée au réseau de nervures des feuilles de cette espèce (Lapeyronie, 1959a, b). On note, en effet, que le classement des points expérimentaux en fonction du rayonnement réellement absorbé par l'échantillon permet de regrouper de façon beaucoup plus satisfaisante les résultats expérimentaux sur la figure 10.

Le rayonnement absorbé $\Delta \Phi$ est calculé suivant l'équation [1]. MC est obtenue à partir des données pondérales, et la pente $\alpha$ est donnée par la cinétique thermique.

II est donc essentiel pour ce type de plante comportant des nervures importantes et hétérogènes de choisir des zones de mesure aussi homogènes que possible.

\section{DISCUSSION ET CONCLUSION}

L'objectif fixé au départ était de développer un appareillage portable utilisable en particulier dans les conditions naturelles pour déterminer la masse surfacique en eau chez les espèces à comportement hydrique contrasté (espèces anisohydrique et isohydrique).
L'appareillage présenté dans cette étude répond bien à cet objectif. Nous avons en effet montré dans le cadre de plusieurs campagnes de mesures que cet appareillage détermine les masses en eau surfacique avec une précision acceptable aussi bien en condition contrôlée que dans les conditions du champ. II est apparu toutefois que dans le cas des feuilles présentant des nervures saillantes et réparties de façon hétérogène, des précautions dans le choix des zones d'échantillonnage doivent être prises. D'un point de vue théorique, il serait préférable d'utiliser de la lumière bleue, mais il faudra d'abord vérifier que l'amélioration de la qualité de la mesure est notable.

En conclusion, le dispositif actuel mis au point est opérationnel pour des mesures au champ. L'appareillage est portable, relativement peu onéreux. La mesure est rapide et suffisamment précise pour mettre en évidence de faibles différences de masse en eau surfacique des feuilles. Cette méthode peut être utilisée pour contrôler les variations d'hydratation des feuilles au champ et pourrait servir à l'établissement de critères pour le pilotage de l'irrigation. 


\section{RÉFÉRENCES}

Amiro BD, Thurtell GW, Gillespie TJ (1983) A small infrared thermometer for measuring leaf temperature in leaf chambers. J Exp Bot 34, 148, 15691576

Baldy C, Konate JM, Olufayo A (1993) Résistance à la sécheresse du sorgho-grain en climat méditerranéen et gestion optimale d'une quantité d'eau limitée. Sécheresse 2, 4, 85-93

Buriol GA, Santibanez F, Menoux Y, de Parcevaux S, Bertolini JM (1984a) Détermination de la masse d'eau et des propriétés optiques d'une feuille à partir de modifications de son bilan énergétique. I. Bases théoriques de la méthode et technique de mesure. agronomie 4, 493-500

Buriol GA, Menoux $Y$, de Parcevaux S (1984b) Détermination de la masse d'eau et des propriétés optiques d'une feuille à partir de modifications de son bilan énergétique. II. Applications en conditions artificielles et naturelles. agronomie 4, 501-506

Graham MED, Thurtell GW, Kidd GE (1989) Calibration of a small infrared sensor for measuring leaf temperature in the field: non-steady-state conditions. Agr For Meteorol 44, 295-305

Kalma JD, Alksnis H, Laughlin GP (1988) Calibration of small infrared surface temperature transducers. Agr For Meteorol 43, 83-98

Katerji N, Itier B, Ferreira I (1988) Étude de quelques critères indicateurs de l'état hydrique d'une culture de tomate en région semi-aride. agronomie 8,425 433

Katerji N, Schoch PG, Rimgoto P, L'Hotel JC (1990) Diagnostic des périodes de contrainte hydrique chez des plantes d'aubergine cultivées en serre, au moyen des microvariations des tiges. agronomie 10, 541-549

Katerji N, Van Horn JW, Hamdy A, Karam F, Mastrorilli $M$ (1994) Effect of salinity on emergence and on water stress and early seedling growth of sunflower and maize. Agricult Water Manag (sous presse)

Kaul R (1969) Relations between water status and yield of some wheat varieties. Z Pflanzenzüchtg 62 , 145-154

Lapeyronie A (1959a) La capacité en eau de limbes foliaires de graminées. CR Acad Sci 248, 2379 2381

Lapeyronie A (1959b) Inversion du gradient de capacité en eau dans le limbe foliaire de graminées. $C R$ Acad Sci 248, 3205-3207

Mastrorilli M, Losavio N, Rana G, Katerji N (1993) Comparison of water stress indicators for soybean. Irrigation of horticultural crops. Act Hort 335

de Parcevaux S (1963) Transpiration végétale et production de matière sèche. Ann Agron 14, 665-742

de Parcevaux S (1972a) Contribution à l'étude des échanges gazeux au niveau des feuilles. Thèse doct État, faculté d'Orsay, Paris-Sud, $86 \mathrm{p}+$ annexes

de Parcevaux S (1972b) Aspects biophysiques des échanges entre les feuilles et le milieu environnant. Oecol Plant 7, 371-401

de Parcevaux S, Perrier A (1973) Bilan énergétique de la feuille. Application de l'étude des cinétiques de température à la détermination des résistances aux flux gazeux. In: Réponse des plantes aux facteurs climatiques (RO Saltyev, ed), UNESCO, Paris, 127135

Roy J (1980) Comportement photosynthétique et hydrique de la feuille chez Dactylis glomerata $\mathrm{L}$. Adaptation phénotypique et génotypique à la sécheresse. Thèse, univ sci techn Languedoc, Montpellier

Santibanez F (1974) Étude des variations de la teneur en eau des feuilles au moyen d'une méthode rapide et non destructive. Thèse, univ Paris-VII, $78 \mathrm{p}$

Tardieu F, Katerji N, Bethenod O (1990) Relations entre l'état hydrique du sol, le potentiel de base et d'autres indicateurs de la contrainte hydrique chez le maïs. agronomie 10, 617-626 